\title{
Cardiac Analysis and Classification of ECG Signal using GA and NN
}

\author{
Naval Kishore \\ M. Tech, Scholar (ECE) \\ RBIEBT, Mohali, Punjab, India
}

\author{
Sukhmanpreet Singh \\ Assistant Professor (ECE) \\ RBIEBT, Mohali, Punjab, India
}

\begin{abstract}
Electrocardiogram represents electrical activity of the compassion. Sinus tachycardia and Sinus Bradycardia are among the most common ECG abnormality. Millions of ECGs are in use for the diagnosis of various lesions of patients, where ECG can give a lot of information regarding the abnormality in the concerned patient; ECGs are analyzed by the physicians and interpreted depending upon their knowledge. The understanding may vary by physician to medical doctor. Hence, this labor is all about the mechanization and consistency in the analysis of the ECG signals so that they must be diagnosed and interpreted accurately irrespective of the physician. This would help to create an early action intended for the problems and many lives might be saved. Many works have been done previously but this work presents Electrocardiogram (ECG) classification to diagnose patient's condition. For classification of such Difficult to Diagnose Signals, P-Wave, PR-Interval, QRS Interval, ST Interval, T- Wave etc, analysis of each Input pulse used to train the neural network and features are obtained using Genetic Algorithm. Output of the neural network gives weight factors of each signal to create a data set. Electrocardiogram (ECG) PQRSTU-waveforms time intervals and weight factors and prediction of particular decease infection or state of a patient condition saved in database. A software program is written in MATLAB 7.10.Corresponding output-datasets indicates related disease and predict the causes. The results show a considerable improvement in conditions of FRR, FAR and accuracy of image retrieval.
\end{abstract}

\section{Keywords}

ECG Signal,Neural Network, Genetic Algorithm, T-Cardia, B-Cardia.

\section{INTRODUCTION}

Noise detection in the ECG signal is the crucial issue in the medical field [1]. For accurate measurement of ECG signal noise reduction is very important [2]. The main aim of

ECG enhancement technique is to reduce the noise and separate the ECG signals so that accurate results can be obtained. The application of ECG signal in medical field is to recognize the ECG signal and then classify the disease according to ECG output [3, 4]. The structure of ECG signal consists of $\mathrm{P}, \mathrm{QRS}$ complex and $\mathrm{T}$ waves [5]. If $0 \in$ $(P, Q, R, S, T)$ Then each component can be represented as below where $\mathrm{Q}$ is height of curve:

$$
\begin{gathered}
\text { P wave: } \mathrm{Q}_{\mathrm{a}} \mathrm{r}-\left(\frac{y-\tau_{p}}{\sqrt{2 \tau_{p}}}\right)^{2} \\
\text { Q wave: } \mathrm{Q}_{\mathrm{a} 1} \mathrm{r}-\left(\frac{y-\tau_{p 1}}{\sqrt{2 \tau_{p 1}}}\right)^{2}+\mathrm{Q}_{\mathrm{a} 2} \mathrm{r}-\left(\frac{y-\tau_{p 2}}{\sqrt{2 \tau_{p 2}}}\right)^{2} \\
\text { R wave: } \mathrm{Q}_{\mathrm{T}} \frac{d e}{d t}\left(\frac{y-\tau_{p}}{\sqrt{2 \tau_{p}}}\right)^{2}
\end{gathered}
$$

$$
\begin{aligned}
& \text { S wave: }-\mathrm{Q}_{\mathrm{d}} \mathrm{e}^{-\left(\frac{y-\tau_{p}}{\sqrt{2 \tau_{p}}}\right) 2} \\
& \text { T wave: } \mathrm{Q}_{\mathrm{y}} \mathrm{e}^{-\left(\frac{y-\tau_{p}}{\sqrt{2 \tau_{p}}}\right)}
\end{aligned}
$$

Detection of QRS is very important w.r.t other waves. But it is very difficult task because of presence of various noises. The noises which may corrupt original ECG signal are:

\subsection{Noise due to muscle contraction \\ (Electromyography interference)}

The power spectrum of this noise is given by

$$
\bar{V}_{n}^{2}=4 k T R
$$

Where $k$ is the Boltzmann's constant, $T$ is the temperature, and $R$ is the resistance

\subsection{Line Interference}

The manifestation of power line noise can be modeled by:

$$
n_{60 H z}(t)=A \sin (2 \pi \cdot 60+\Omega) .
$$

That is why noise removal in QR signal is must. ECG also used in heart disease classification that is why ECG signal must be noise free $[6,7]$. The ECG morphology used for heart detection [8]. The ECG, over a solitary cardiovascular cycle, has a trademark morphology containing a $\mathrm{P}$ wave, a QRS complex and a $\mathrm{T}$ wave. The typical ECG setups are made out of waves, edifices, sections and interims recorded as voltage (on a vertical pivot) against time (on a flat hub) $[9,10]$. A solitary waveform starts and finishes at the gauge. At the point when the waveform proceeds past the benchmark, it changes into another waveform [11]. Two or more waveforms together are known as a complex. A level, straight, or isoelectric line is known as a fragment. A waveform, or perplexing, associated with a section is called an interim. All ECG tracings over the pattern are portrayed as positive redirections. Therefore, ECG signal must be clearly shown and needs to be noise free [12]. The development of exact and fast method intended for regular ECG feature removal that is of chief significance, mostly used for the assessment of long recordings. ECG gets ruined because of a variety of the artifact [13]. 


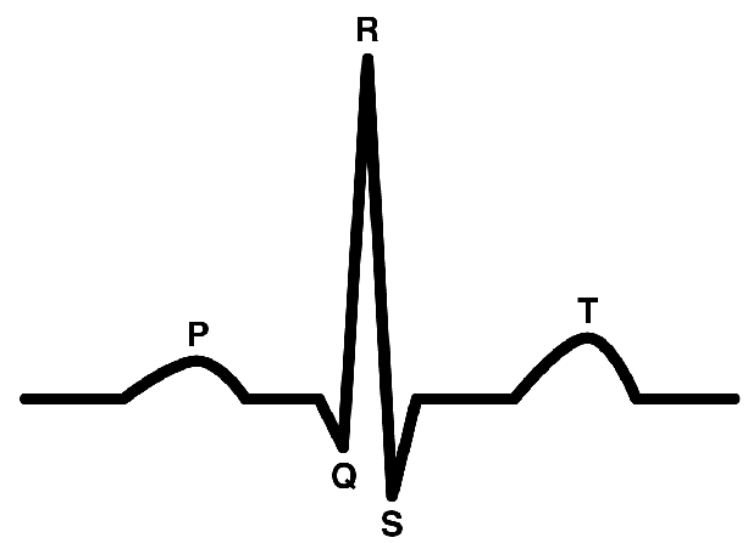

Fig 1: ECG Waveform

In proposed work two diseases like B-Cardia and T-Cardia will be classified using combination of neural network and genetic algorithm. Genetic algorithm will work as feature reduction technique and neural network will work as disease classification technique. The rest of the paper is organized as:

\section{GENETIC ALGORITHM}

Harik analyzed the growth and decay of a particular gene in the population as one dimensional random walk. As the GA progresses, genes fight with their competitors and their number in the population can go up or down depending on whether the GA makes good or bad decisions. These decisions are made implicitly by the GA when selection takes place. The next section explores the effects of this decision making [19].

\subsection{Selection}

Proportionate reproduction describes a group of selection schemes that choose individuals according to their objective function values $\mathrm{f}$.

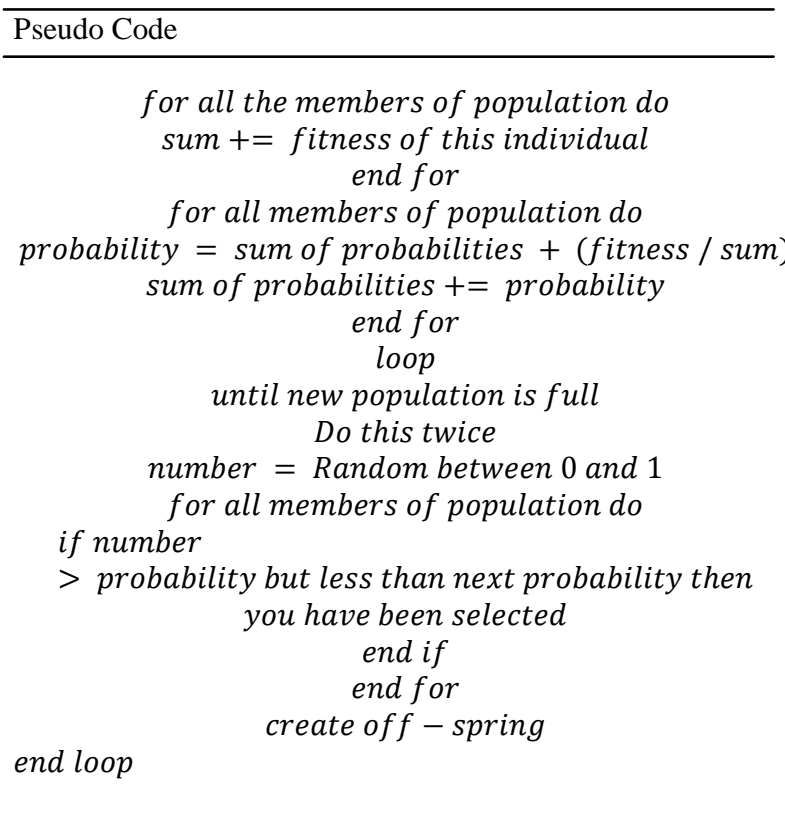

\subsection{Crossover}

The role of crossover in the GA is to combine bits and pieces from fit solutions.

Pseudo Code find a crossover point according to the

Crossover probability.

for $i=0$ to crossover point do

child $A$ gene $[i]=$ parent $A$ gene $[i]$

child $B$ gene $[i]=$ parent $B$ gene $[i]$

end for

for $i=$ crossover point to chromosome length do child $A$ gene $[i]=$ parent $B$ gene $[i]$ child $B$ gene $[i]=$ parent $A$ gene $[i]$ end for

return children

\subsection{Mutation}

Similar to crossover, mutation is analogous to biological mutation and used to maintain genetic diversity from one generation to the next. The aim of mutation in GAs is to allow the algorithm to avoid local minima by preventing the population of chromosomes from becoming too similar to each other, thus slowing or even stopping evolution.

Pseudo Code

for $i=1$ to chromosome length do generate a random number $\mathrm{Nr}$

if $\mathrm{Nr}$ fi mutation probability then child $A$ gene $[i]=$ random value

child $B$ gene $[i]=$ random value end if

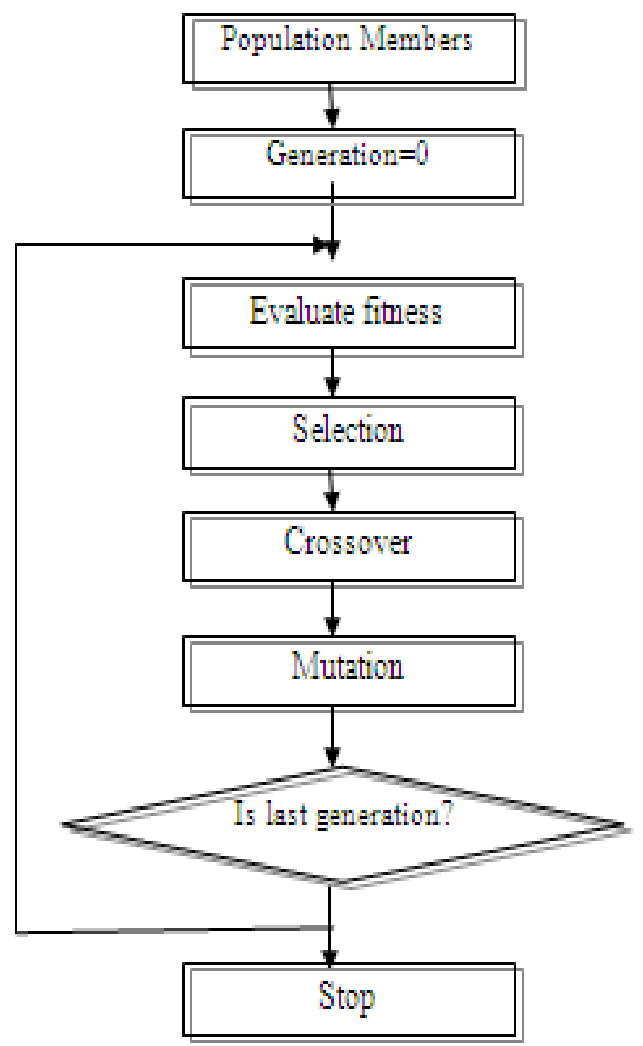

Fig 2: Genetic Algorithm Flowchart 


\section{NEURAL NETWORK}

A neural network is type of connected system in which various neurons are connected to each other. The neural network does not work in linear phase rather it works in parallel manner. Basic architecture of neural network is represented as follows [20]

The perceptron is the simplest neuron with the help of which working will be discussed as following [21]:

Pseudo Code

\section{Receive inputs}

Input $i 1.12 \ldots$. in

Assign Weights to inputs

$I 1 w 1, i 2 w 2$...inwn.

Sum of all the inputs

$I 1 w 1+i 2 w 2+\cdots \ldots+i n w n$

Generate Output

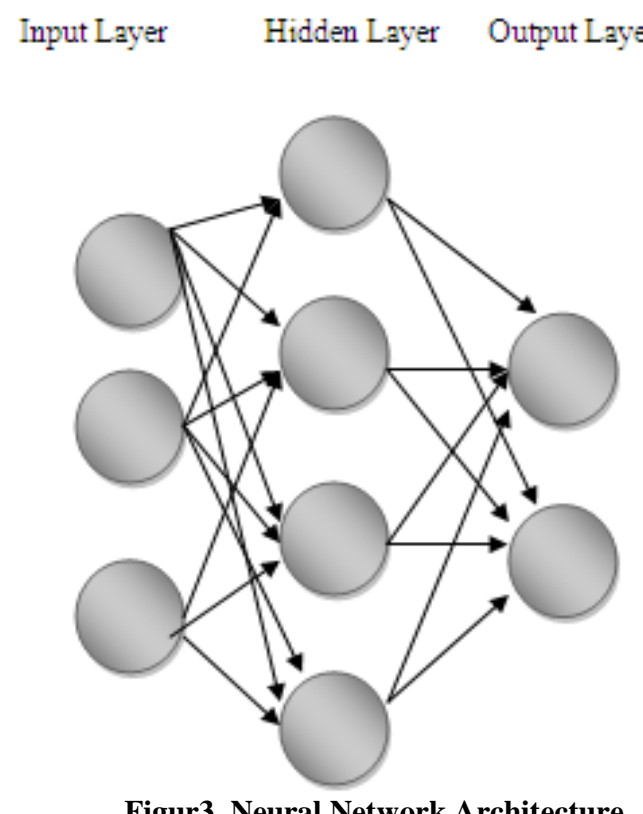

\section{COMPUTATION STUDY}

As the ECG signals are used for detecting the cardiac diseases and the improvement in ECG feature extraction has become the chief importance for diagnosing the long recording. An ECG signal is a graphical representation of the cardiac activity for computing the cardiac diseases and to check the abnormalities in the heart. The problem of this research work is to classify the disease dataset using Genetic algorithm and train the Neural Network on the basis of the features extracted and also to test the image on the basis of the features at the database and the features extracted of the image to be tested. Our research is based on studying the implemented approaches in the ECG diseases and then to propose a new method /algorithm for classification of Sinus Bradycardia and
Tachycardia depend on neural network and the genetic algorithm.

The study is implemented by using the various parameters like false acceptance Ratio (FAR), false rejection rate (FRR) and Accuracy. A system's FAR typically is the ratio of the number of false acceptances to the number of identification attempts. FRR typically is the ratio of the number of false rejections divided by the number of identification attempts and accuracy defines how close a measured value is to the actual value. The steps above shown in the flowchart explained the phase that has been accomplished.

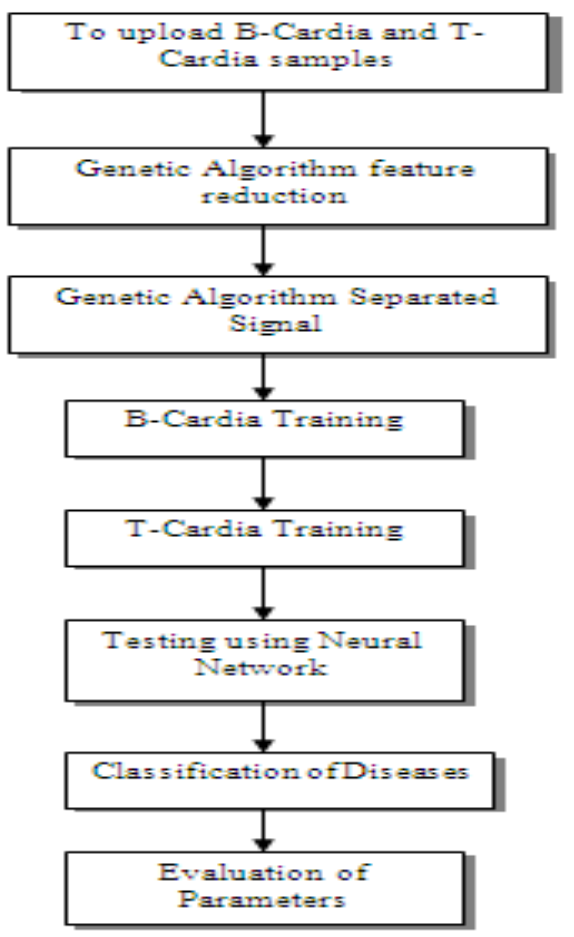

Fig 4: Proposed Flowchart

\section{RESULT}

The whole implementation has been done in MATLAB environment using GA and NN algorithms.

Table 1: Values of different parameters

\begin{tabular}{|l|l|l|l|l|}
\hline $\begin{array}{l}\text { Sample } \\
\text { s }\end{array}$ & Disease & FAR & FRR & $\begin{array}{l}\text { Accurac } \\
\mathbf{y}\end{array}$ \\
\hline $\mathbf{1 0 4}$ & $\begin{array}{l}\text { Techacardi } \\
\text { a }\end{array}$ & $\begin{array}{l}0.01935 \\
4\end{array}$ & $\begin{array}{l}0.0004605 \\
4\end{array}$ & 99.9346 \\
\hline $\mathbf{1 0 6}$ & $\begin{array}{l}\text { Bradacardi } \\
\text { a }\end{array}$ & 0.00973 & 0.00034 & 99.9562 \\
& $\begin{array}{l}\text { Bradacardi } \\
\text { a }\end{array}$ & 0.01001 & 0.0003566 & 99.9543 \\
\hline $\mathbf{1 2 3}$ & $\begin{array}{l}\text { Techacardi } \\
\text { a }\end{array}$ & 0.01564 & 0.0002727 & 99.9571 \\
& 4 & 7 & \\
\hline $\mathbf{2 1 9}$ & & & & \\
\hline
\end{tabular}

FAR is the measure of the false acceptance rate values in the propsoed work. Its value must be low in order to have good efficiency of the proposed implemented algorithm. From 
above figure 5: it has been concluded that FAR values for proposed samples of database are $.0193, .0097, .0156$ and .0100 .

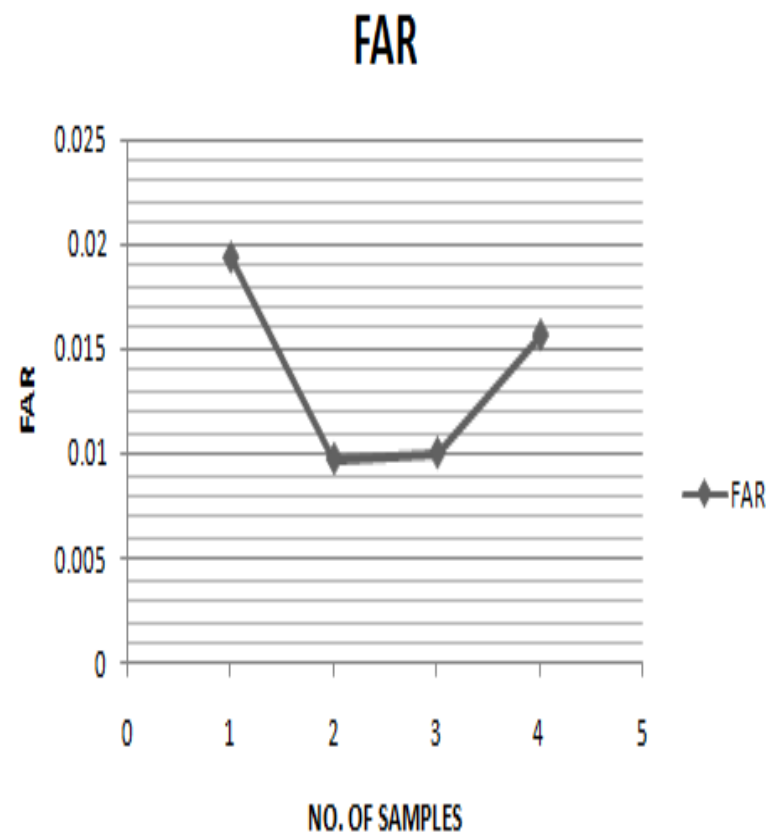

Fig 5: FAR Values

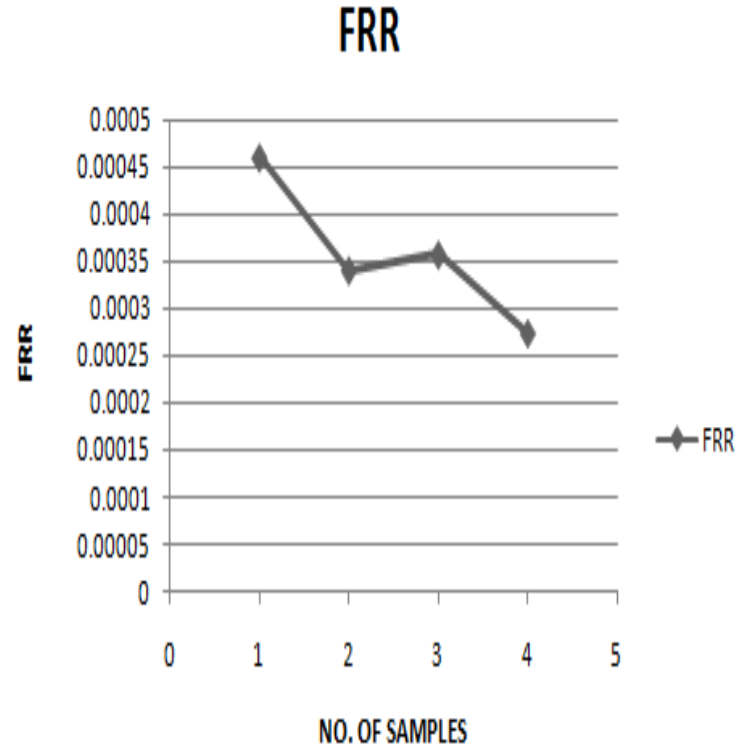

Fig 6: FRR Values

FRR is the measure of the false rejection rate values in the propsoed work. Simillar to FAR, the FRR values must be low in order to have good efficiency of the proposed implemented algorithm. From above figure $6:$ it has been concluded that FRR values for proposed samples of database are .00046, .00034 .00035 and .000272 .

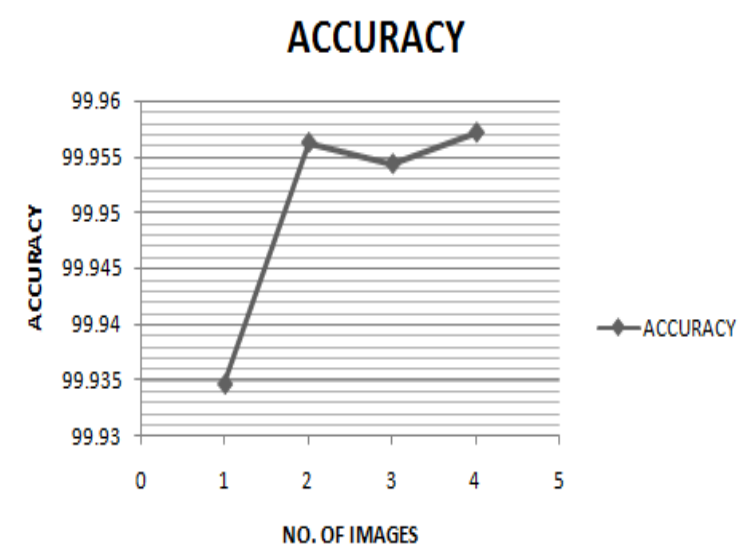

Fig 7: Accuracy Values

The above figure shows the accuracy in terms of FAR and FRR. It can be written as:

$$
\operatorname{Accuracy}(\%)=(100-(F A R(\%)+F R R(\%)) / 2)
$$

Above figure shows the average accuracy rate of $99.96 \%$ using neural network and genetic algorithm. Hence implemented algorithm is better with respect to accuracy.

Table 2: Performance Comparison

\begin{tabular}{|c|c|c|}
\hline Approach & FAR & FRR \\
\hline $\begin{array}{c}\text { Proposed } \\
\text { Approach }\end{array}$ & .0123 & .0038 \\
\hline $\begin{array}{c}\text { Chiu et.al (DB-1) } \\
{[22]}\end{array}$ & .83 & .86 \\
\hline $\begin{array}{c}\text { Chiu et.al (DB-2) } \\
{[22]}\end{array}$ & 12.50 & 5.11 \\
\hline
\end{tabular}

Table 2 shows the performance comparison of our proposed method with several existing method, where our proposed method has a better performance in FAR and FRR. The reason for good FAR and FRR is that the proposed method used combination of genetic algorithm and neural network.

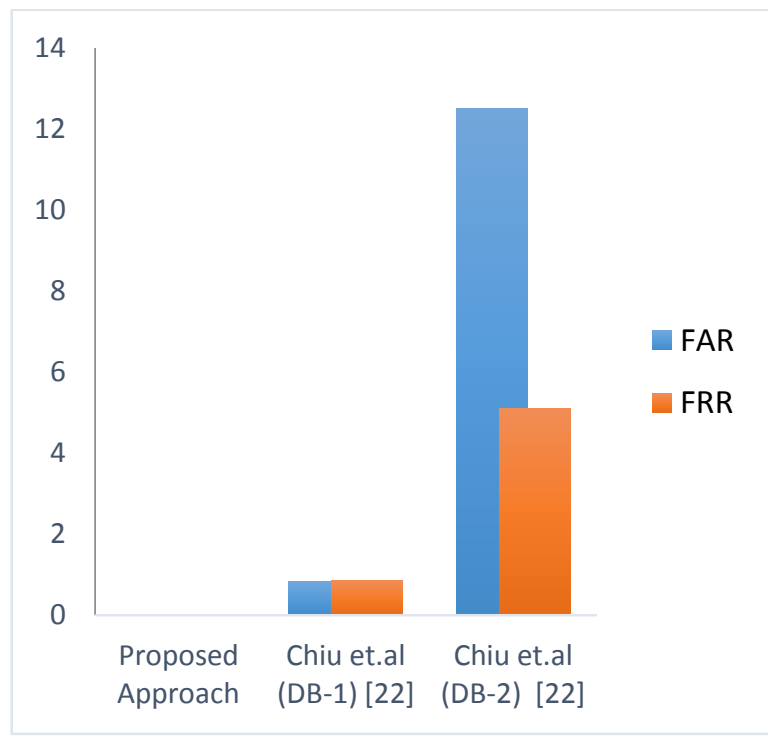

Figure 8. Comparison Graph 


\section{CONCLUSION}

Clinical databases have accumulated large quantities of information about patients and their medical conditions. The term Heart disease encompasses the diverse diseases that affect the heart. Heart disease is the major cause of casualties in the world. The term Heart disease encompasses the diverse diseases that affect the heart. Heart disease kills one person every 34 seconds in the United States. Record set with medical attributes was obtained from any Repository. With the help of the dataset, the patterns significant to the heart attack prediction can be extracted. The ECG Disease Detection System based on Genetic Algorithm and Neural Network, in which detection is based on three parameters accuracy, FAR and FRR is presented in this research. In the end, classification has been done between T-Cardia and BCardia diseases. Simulation results shows that the obtained values of FAR, FRR and accuracy for proposed tested image has been found out to be FAR=.0100, FRR $=.00010$ and accuracy $=99.97 \%$, that are the satisfactory results. Future scope lies in the use of other classifiers like SVM that has multidimensional data and use of feature reduction algorithms genetic algorithm, so that accuracy rate can be enhanced.

\section{REFERENCES}

[1] P. de Chazal, M. O'Dwyer, and R. B. Reilly, "Automatic Classification of Heartbeats Using ECG Morphology and Heartbeat Interval Features," IEEE Trans. on Biomedical Engineering, Vol. 51, No. 7, pp.1196-1206, July 2004.

[2] C. Alexakis, H. O. Nyongesa, R. Saatchi, N. D. Harris, C. Davis, C. Emery, R. H. Ireland, and S. R. Heller, "Feature Extraction and Classification of Electrocardiogram (ECG) Signals Related to Hypoglycaemia," Computers in Cardiology, Vol. 30, pp.537-540, 2003.

[3] I. Romero and L. Serrano, "ECG Frequency Domain Features Extraction: A New Characteristic for Arrhythmias Classification," Proc. Int'1. Conf. on Engineering in Medicine and Biology Society (EMBS'2001), pp.2006-2008, October 2001.

[4] P. de Chazal and R. B. Reilly, "A Comparison of the ECG Classification Performance of Different Feature Sets," Computers in Cardiology, Vol. 27, pp.327-330, 2000 .

[5] Y. H. Hu, W. J. Tompkins, J. L. Urrusti, and V. X. Afonso, "Applications of Artificial Neural Networks for ECG Signal Detection and Classification," Journal of Electrocardiology, Vol. 26. (Suppl.), pp.66-73, 1994.

[6] S. Behbahani and N. J. Dabanloo, "Detection of QRS complexes in the ECG signal using Multiresolution wavelet and thresholding method" IEEE Computing in Cardiology, pp. 805-808, 2011.

[7] Hu, S.; Shao, Z.; Tan, J. A real-time cardiac arrhythmia classification system with wearable electrocardiogram. In Proceedings of the IEEE International Conference on Body Sensor Networks (BSN), Piscataway, NJ, USA, 23 May 2011; pp. 119-124.

[8] Yu, S.-N.; Chen, Y.-H. Electrocardiogram beat classification based wavelet and probabilistic neural network. Pattern Recogn. Lett. 2007, 7, 1142-1150.
[9] Rodriquez, J.; Gorii, A. Real-time classification of ECGs on a PDA. IEEE Trans. Inf. Technol. Biomed. 2005, 3 , 23-34.

[10] Christov, I.; Gomez-Herrero, G. A comparative study of morphological and time-frequency ECG descriptors for heartbeat classification. Med. Eng. Phys. 2006, 2, 876887.

[11] S.Sumathi, Dr.M.Y. Sanavullah, "Comparative Study of QRS Complex Detection in ECG Based on Discrete Wavelet Transform," Int. Journal of Recent Trends in Engineering, Vol 2, No. 5, November 2009, pp.273-277

[12] Krimm, H.; Brooks, D.H. Feature-based segmentation of ECG signals. In Proceedings of the IEEE International Symposium on Time-Frequency and Time-Scale Analysis, Paris, France, 18-21 June 1996; pp. 97-100.

[13] Pergamon, P. Comprehensive Electrocardiology: Theory and Practice in Health and Disease, 1st ed.; Pergamon Press: New York, NY, USA, 1989; Volume 1-3.

[14] A. Dallali, A. Kachouri and M. Samet, "Fuzzy C-means clustering, neural networks, WT, \& HRV for classification of cardiac arrhythmia" in ARPN Journal of Engineering and Applied Sciences, October 2011, ISSN 1819-6608, VOL. 6, NO. 10, pp. 112-118.

[15] Argyro Kampouraki, George Manis, and Christophoros Nikou, "Heartbeat Time Series Classification with Support Vector Machines," IEEE Trans. on Information Technology in Biomedicine, Vol. 13, No. 4, July 2009 pp. 512- 518 .

[16] Dusit Thanapatay, Chaiwat Suwansaroj, Chusak Thanawattano, "ECG beat classification method for ECG printout with Principle Components Analysis and Support Vector Machines", International Conference on Electronics and Information Engineering, Volume 1, 2010, pp. $72-75$

[17] Manpreet Kaur, A.S.Arora, "Unsupervised Analysis of Arrhythmias using K-means Clustering," International Journal of Computer Science and Information Technologies, Vol. 1 (5) , 2010, pp. 417-419.

[18] Emina Alickovic, Abdulhamit Subasi ," Medical Decision Support System for Diagnosis of Cardiovascular Diseases using DWT and kNN".

[19] J. Sarma and K. De Jong, "An Analysis of Local Selection Algorithms in a Spatially Structured Evolutionary Algorithm," Proc. Int'l Conf. on Genetic Algorithms (ICGA-97), pp. 181-186, 1997.

[20] S. W. Moon and S. G. Kong, "Pattern Recognition with Block-based Neural Networks," Proc. Int'l J. Conf. on Neural Networks (IJCNN-2002), pp.992-996, May 2002 .

[21] S. G. Kong, "Time Series Prediction with Evolvable Block-based Neural Networks," Proc. Int'l J. Conf. on Neural Networks (UCNN-2004), July 2004.

[22] Chuang-Chien Chiu, Chou-Min Chuang, And Chih-Yu Hsu, Int. J. Wavelets Multiresolut Inf. Process. 07, 341 (2009). DOI: 10.1142/S0219691309002957 